\title{
An empirical study of vibration based bearing fault diagnosis methods
}

\author{
Kurt Pichler ${ }^{1}$, Ted Ooijevaar ${ }^{2}$, Clemens Hesch $^{1}$, Christian Kastl ${ }^{1}$ \\ ${ }^{1}$ Linz Center of Mechatronics GmbH, Altenberger Straße 69, 4040 Linz, Austria \\ ${ }^{2}$ DecisionS, Flanders Make, Gaston Geenslaan 8, 3001 Leuven, Belgium
}

\begin{abstract}
This paper presents an empirical study in which universally applicable fault diagnosis methods are used to evaluate vibration data of bearings. The data were acquired on two different test beds: a gear box test bed containing various bearings at different health states, and an accelerated life time (ALT) test bed to degrade a bearing and introduce an operational fault. Features are extracted from the raw data of two different accelerometers and used to monitor the actual health state of bearings. For that purpose, feature selection and classifier training is performed in a supervised learning approach. For testing the proposed approach, cross validation is applied to the data. The results of the gearbox test bed show that the classification accuracy data increases with the revolution speed of the bearing. Furthermore, the data of a high-end sensor allow higher classification accuracy than the data of a lowcost sensor. The results of the ALT test bed show that the same features that were identified in the gearbox test start to change significantly when the bearing degrades.
\end{abstract}

Keywords: Vibration Analysis, Fault Detection, Bearing, Feature Engineering, Machine Learning

\section{Introduction}

Manufacturing companies continuously try to increase their productivity, among others by avoiding machine down times. The latter involves considerable costs because of the resulting loss of turnover. Monitoring the condition of, for instance, bearings and gears, plays a vital role in the maintenance program of rotating machines. Early fault detection could allow to move from a time based preventive maintenance program to a condition based predictive maintenance strategy and reduce unexpected machine downtime and cost.

Vibration based condition monitoring is an established approach that has been employed by industries for many years in their maintenance program. However, up to this day, machine operators often still base their maintenance decisions on data from the periodical and manual inspection of single machines, which does not always result in correct conclusions. The common practice is that vibration measurements are periodically recorded using portable vibration sensors and measurement signals are analysed by an expert to interpret the machine's health condition. This approach can, however, lead to serious misinterpretation, where rapidly growing faults could be missed.
A continuous condition monitoring approach enables an early detection of machine faults. In this way, the machine condition is continuously tracked and total failures can be anticipated in advance, hence allowing appropriate maintenance actions. Despite its advantages, continuous monitoring program is however still not well adopted by industry. Firstly, because it often involves a high investment cost. Although recent advancements in sensor, acquisition and processing hardware have demonstrated cost-effective solutions [1,2], the economic benefit of the investment is still not clear and hard to quantify. Secondly, because many of those systems still require an expert to interpret the analysis results. Finally, also because it is not straight forward to select the most appropriate method for a specific application.

A wide range of vibration based bearing fault detection methods have been proposed in literature $[3,4,5]$. Approaches that utilize time domain features (e.g. crest factor, kurtosis), frequency and cepstral domain features (e.g. envelope analysis, cepstral coefficients) usually assume stationary machine conditions. Other methods such as cyclo-stationary analysis (i.e. second order technique in the frequency domain) and time-frequency domain analysis (e.g. Wigner-Ville distribution, HilbertHuang transform and wavelet transform based features) are more appropriate for non- 
stationary processes. Some of those methods are purely data driven, whereas others use the physical relation between the bearing geometry, the rotational shaft speed and the bearing specific fault frequencies associated to the impulse behavior introduced by bearing faults.

In this paper, we present a purely data-driven method by extracting a large number of features from vibration data and subsequent selection and classification of those features in a supervised learning approach. Test data were acquired at two different bearing test beds: a gearbox setup that can be equipped with bearings of different degradation status, and a simple rotating shaft with a bearing under certain radial loads for accelerated degradation. The following chapters describe the data analysis method, the experimental setup and the evaluation results.

\section{Description of the method}

The fault diagnosis approach adopted here is a purely data-driven one, i.e. it incorporates no physical knowledge about the monitored system. That makes it on one hand more flexible and applicable to many kinds of systems, machines or components. On the other hand, incorporating extra knowledge usually improves the diagnostic ability of a condition monitoring system.

The first step of the proposed procedure is to extract a large number of features (for instance [6-16]) from the raw vibration signal. These features include simple statistical measures like standard deviation or kurtosis as well as more advanced features in time, (time-)frequency, quefrency and wavelet domain.

Subsequently, the dimensionality of the feature space is reduced to avoid the curse of dimensionality. Therefore, the significant features are identified by feature selection procedures as described in [17]. In particular, a standard forward selection filter algorithm selecting one feature per step was applied. As selection criterion in each step we adopted robust distance measures like Dy-Brodley measure [18] or Mahalanobis distance [19]. Feature selection is stopped when the relative gain of the selection criterion falls below $1 \%$. In all of our tests, both distance measures selected the same features until the stopping criterion was reached.

After feature extraction and selection, a classifier in the feature space is trained. For that purpose we use linear or quadratic normal Bayes classifiers [20]. These classifiers assume normally distributed classes and apply Bayes decision rule to those classes. In the linear case, the co-variances of the classes are assumed to be equal. The validity of using normally distributed classifiers was checked by normal probability plots of the feature vectors (see two examples in Fig. 1).

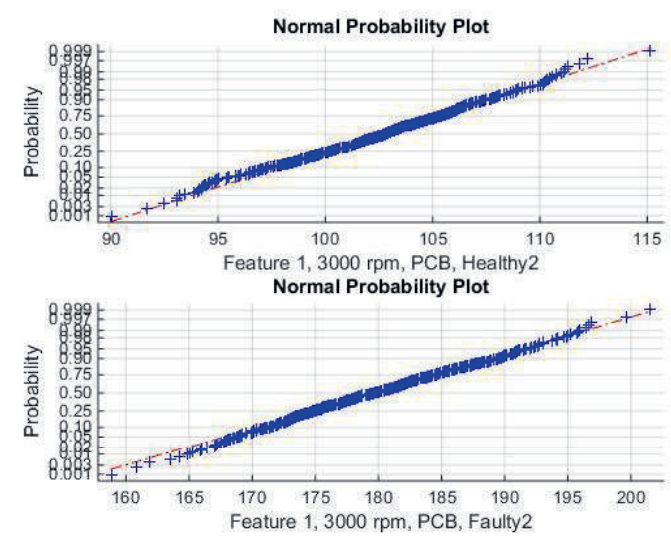

Fig. 1: Normal probability plots of Feature 1, $3000 \mathrm{rpm}$ data, PCB sensor, states Healthy2 and Faulty2

The supervised learning approach implies that the method depends on having a sufficient amount of annotated training data for all states (failure modes) to be monitored. There are also classifiers for one-class classification (also referred to as novelty detection) available [21]. However, those techniques detect only deviation from a nominal state and are thus prone to over detection due to changing operation modes. Furthermore, the feature selection process depends on having an annotated data set as well.

The process of evaluating a new data sample is straightforward: the selected features are extracted from the raw signals, and the classifier is applied to those features. Since many classifiers are able to deliver class membership probabilities, it is generally possible to determine instances lying between two distinct states. However, here we restrict the evaluation to crisp class decisions by detecting the maximum class probability for each observation.

Given annotated training data, the whole process of feature extraction, feature selection and classification can be fully automated. The more useful information the training data contain, the better the resulting feature subset and classifier will be. In this context, information means different states, rotation speed, repeated measurements with different samples of the same bearing type, and so on.

\section{Experimental Setup}

Two types of experiments have been performed: (i) an accelerated life time test $(A L T)$ of a ball bearing on a single shaft drive 
train set-up and (ii) a test on a more complex gearbox set-up including bearings with various faults. These setups were provided by the scientific partner FLANDERS MAKE in Leuven. The ALT allows to create an operational fault in a bearing. This test differentiates from other studies in the fact that they are often limited to artificially induced faults. Moreover, the fault evolution and accumulation can be monitored during the ALT.

The experimental set-up used to perform the accelerated life time test is shown in Fig. 2 . The set-up comprises of a single shaft with a test bearing. The shaft is supported with the help of a support bearing on each side. A hydraulic cylinder is used to apply a radial load to the test bearing up to a maximum of $10 \mathrm{kN}$. The set-up is driven by a motor at a fixed rotation speed of $1500 \mathrm{rpm}$.

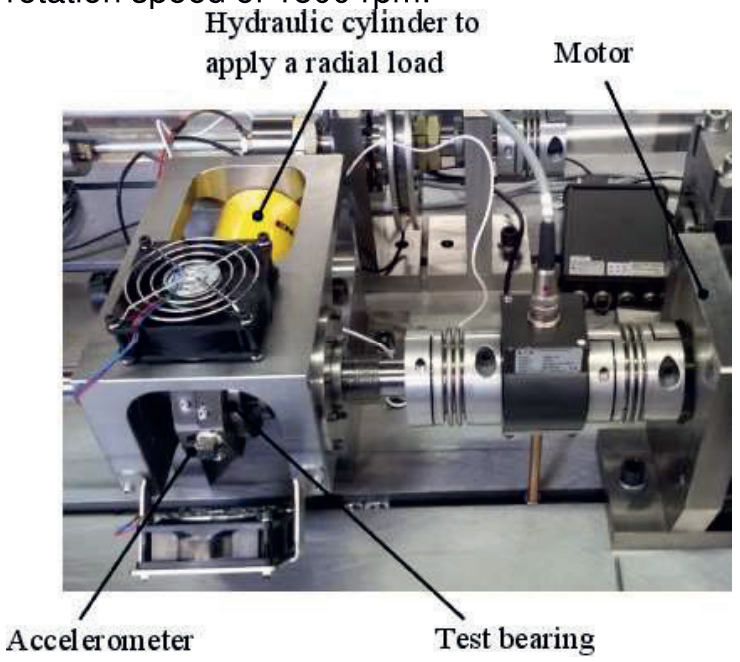

Fig. 2: $\quad$ The drive train set-up used to reduce the life time of a bearing to less than a day, allowing to generate vibration data during the accumulation of an operational bearing fault.

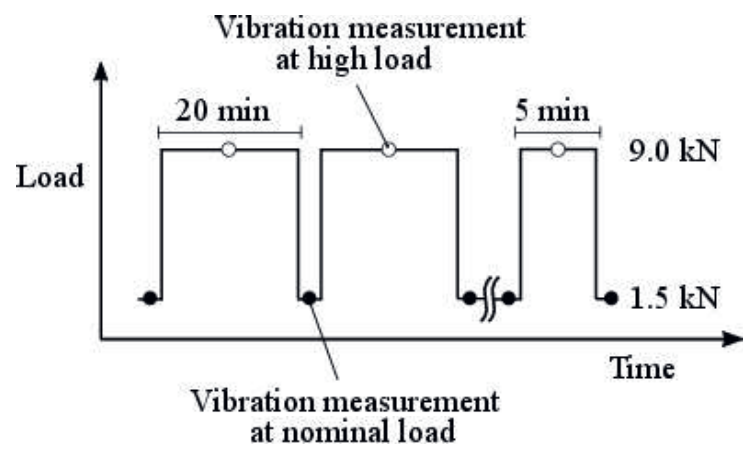

Fig. 3: The load was temporarily increased from $1.5 \mathrm{kN}$ to $9 \mathrm{kN}$ to accelerate the wear of the bearing.

The test procedure is schematically illustrated in Fig. 3. Vibration measurements were performed under a nominal radial load of 1.5
$\mathrm{kN}$ (i.e. $10 \%$ of the dynamic load rating). The radial load was temporarily increased to $9.0 \mathrm{kN}$ (i.e. $65 \%$ of the dynamic load rating) to accelerate the degradation of the bearing. In the beginning the interval was 20 minutes, but this has been reduced as soon as the first indication of an incipient fault was noticed in the measured vibration responses. In total 30 vibration measurements were performed at the nominal $1.5 \mathrm{kN}$ loading condition and 29 vibration measurements at the high $9.0 \mathrm{kN}$ radial load.

The applied radial load, the radial vibrations in the loading direction and the temperature of the bearing housing were measured during the test. The machine vibrations were measured using a piezo film $\mathrm{ACH}-01-03$ accelerometer and digitized at $12.8 \mathrm{kHz}$ by an embedded acquisition platform. In each measurement 20 seconds of data was acquired. The accelerated life time test was stopped when a vibration peak level of $+/-50 \mathrm{~g}$ was reached.

The ALT was performed on a FAG 6205 ball bearing. Before the start of the test a small indentation with a diameter of $230 \mu \mathrm{m}$ was created in the inner race. This indentation is used as a local stress riser and represents a local plastic deformation caused by, for instance, a contamination particle. Subsequently, the ALT was performed for several hours. Although bearings can fail in many different ways, the indentation triggers the bearing to fail in a more repeatable way. The test was stopped when severe rolling contact surface fatigue occurred at the inner race [22]. The start and the end condition of the inner race of the test bearing are shown in Fig. 4.

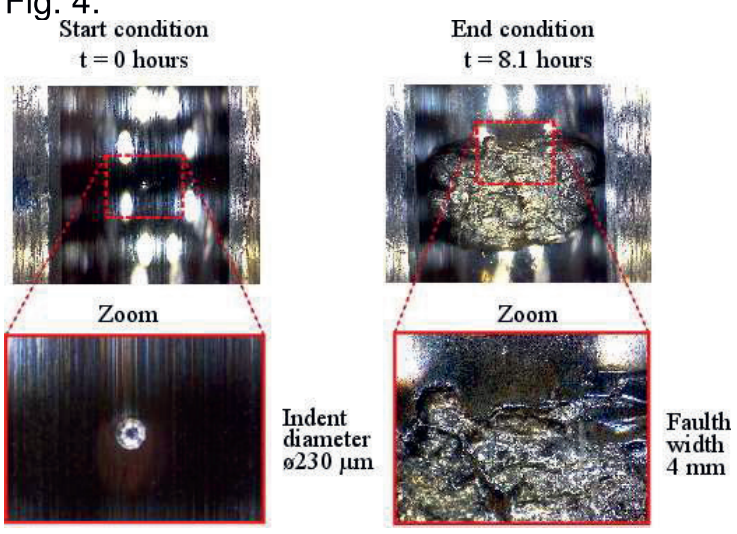

Fig. 4: The indentation at the bearing inner race used as the start condition and the surface fatigue fault at the inner race introduced by the $A L T$.

The second test performed in this study was an industrially representative gearbox setup. Fig. 5 shows a photograph and a schematic top-view of the gearbox setup. The test setup 
consists of (i) an induction electric motor, (ii) a gearbox and (iii) a magnetic brake. The motor is controlled by a variable-frequency-drive (VFD) with either a stationary mode or a transient mode (run-up/run-down). The motor speed can be controlled from 0 to $3000 \mathrm{rpm}$. The gearbox input shaft is connected to the motor, the gearbox output shaft is coupled to the brake. The torque applied to the brake can be adjusted by the controller from 0 to $50 \mathrm{Nm}$. As illustrated in Fig. 5, the gearbox comprises of three-parallel shafts connected through contacting spur gear pairs. Note that the number of gear teeth is indicated in the figure. The input shaft is supported by deep groove ball bearings MB ER-10K, while the other shafts are supported by deep groove ball bearings MB ER-16K. For simulating a healthy or faulty state on the gearbox, the right-side bearing housing that supports the second shaft is equipped either with a healthy or a damaged ball bearing of type FAG 6205-C-TVH.

Two healthy bearings and three faulty bearings with different inner race faults were tested. An indentation fault with a diameter of $490 \mu \mathrm{m}$ was created. Two other bearings with operational faults were created using the ALT setup as described above. The healthy bearings are referred as 'Healthy1' and 'Healthy2', while the faulty bearings are referred as 'Indent', 'Faulty1', 'Faulty2', in the order of increasing severity and are illustrated in Fig. 6.
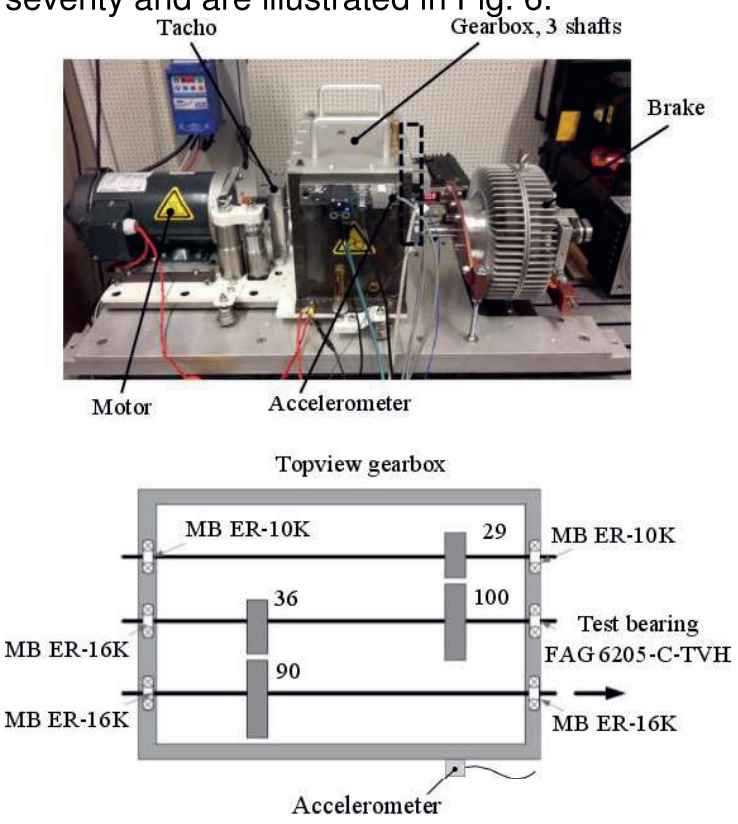

Fig. 5: Gearbox set-up comprising a motor, 3-shaft gearbox and brake to introduce a load.

For each healthy or faulty state, 2 operating conditions were imposed on the gearbox setup, namely two different motor speeds of 1500 and 3000 rpm, respectively. The brake torque was kept constant at $50 \mathrm{Nm}$. Because of the transmission ratio, the rotational speed of the second shaft is $29 / 100$ lower than that of the motor speed, while the torque applied on the second shaft is $36 / 90$ lower than that of the brake torque. Hence, for the imposed operating conditions, the rotational speeds of the second shaft were 435 and $870 \mathrm{rpm}$, while the torque applied to the second shaft was 20 $\mathrm{Nm}$. A high-end PCB accelerometer and a low cost MEMS accelerometer were mounted on the gearbox housing as shown in Fig. 5. The vibration signals were sampled at $50 \mathrm{kHz}$ in 20 seconds data batches.

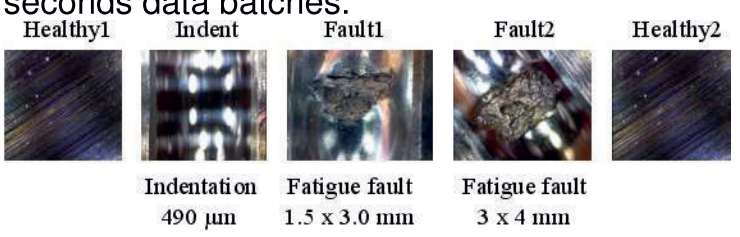

Fig. 6: Five bearing states tested on the gearbox setup comprising two healthy bearings and three faulty bearings with different severities.

\section{Results}

In the ALT the features were extracted from the raw sensor signals in an overlapping sliding window approach (window length 0.2 seconds, overlap 0.1 seconds). That yields in 199 observations out of each 20 second data batch. However, for final evaluation, only the mean value of those 199 observations of a data batch is observed. Unlike the gearbox setup, we had no data of different health states available for feature selection and classifier training in the accelerated life time test. Therefore we were restricted to detect significant changes in the feature values. For that purpose, cumulative sum (CUSUM) control charts [23] were applied to the features. Due to the missing feature selection step, we evaluated the top ranked features of the gearbox test. All of those features increased significantly towards the end of the test run for the $9.0 \mathrm{kN}$ as well as for the $1.5 \mathrm{kN}$ load conditions. For example, feature 20 is depicted in Fig. 7. Due to the increasing feature value, the upper threshold of the CUSUM control chart was exceeded after approximately 7.3 hours in the $9.0 \mathrm{kN}$ case and 7.9 hours in the $1.5 \mathrm{kN}$ case, indicating a failure of the bearing. For control purposes, Fig. 8 shows an arbitrarily chosen feature. The feature shows no significant trend, and the CUSUM control charts don't exceed the thresholds.

Just like in the ALT, the features for the gearbox test were extracted from the raw acceleration signals in an overlapping sliding window approach with a window length of 0.2 
seconds and an overlap of 0.1 seconds, delivering again 199 observations for each 20 second data batch. After extraction of all features, the feature selection algorithm was applied to the $3000 \mathrm{rpm}$ and $1500 \mathrm{rpm}$ motor speed data independently. Furthermore, we did not use all available states for feature selection. We used only the datasets of the states Healthy1, Healthy2 and Faulty2, but not Indent and Faulty1.
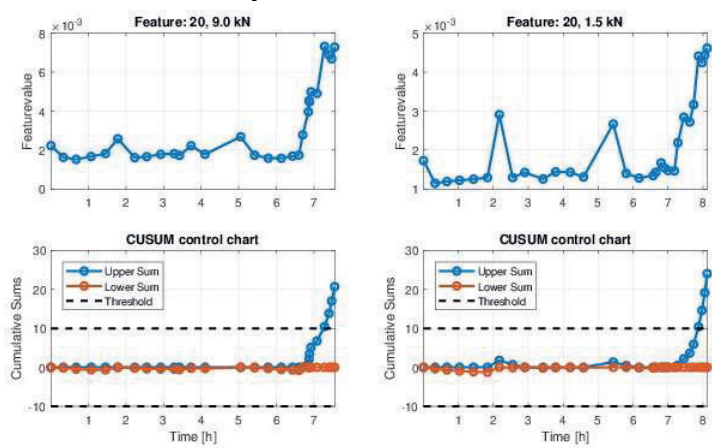

Fig. 7: Feature values and CUSUM control charts for feature 20 in the ALT test for load $9.0 \mathrm{kN}$ (left) and $1.5 \mathrm{kN}$ (right).
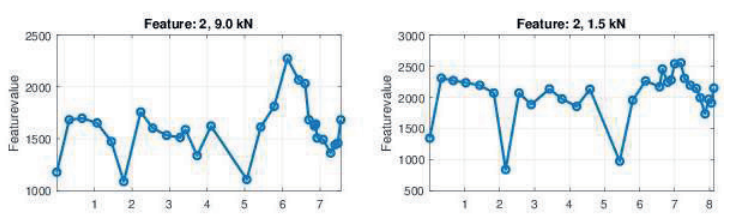

Cusum control chat
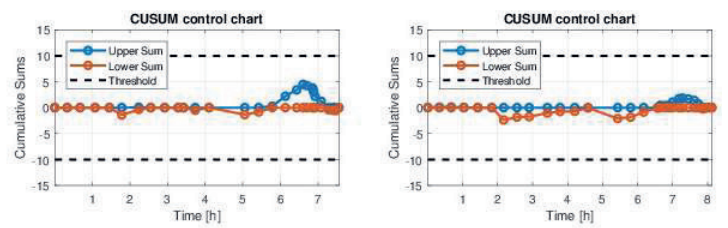

Fig. 8: Feature values and CUSUM control charts for feature 2 in the ALT test for load $9.0 \mathrm{kN}$ (left) and $1.5 \mathrm{kN}$ (right).

We first evaluate the data of the high-end PCB accelerometer. For the $3000 \mathrm{rpm}$ motor speed data, the algorithm selected 3 top ranked features and for the $1500 \mathrm{rpm}$ data it selected 4 top ranked features. However, for a first visual impression we show only the top 2 features for all recorded rotation speeds and all recorded states in a scatter plot in Fig. 9 (3000 rpm) and Fig. 10 (1500 rpm).

The scatterplots indicate already a few possible conclusions:

- Different top features were selected for the different rotation speeds

- The $3000 \mathrm{rpm}$ dataset revealed better separability

- Faulty1 and Faulty2 produced similar feature values
- In the $3000 \mathrm{rpm}$ dataset, the Indentclass lies somewhere in-between the healthy and the faulty states

PCB, 3000 rpm

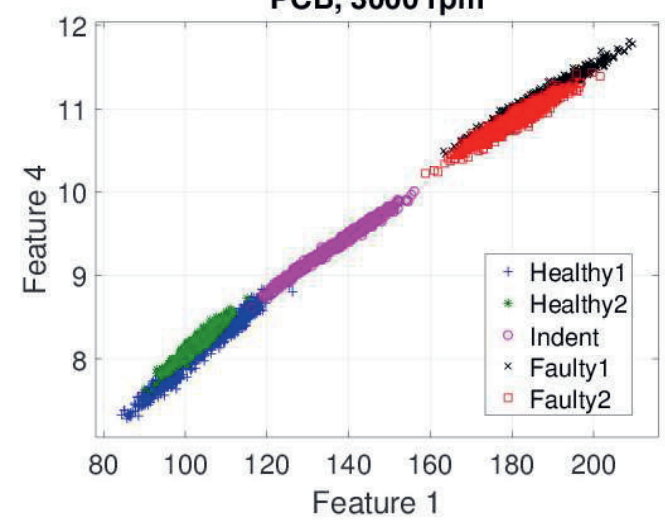

Fig. 9: $\quad$ Scatter plot of top 2 features for the 3000 rpm PCB dataset.

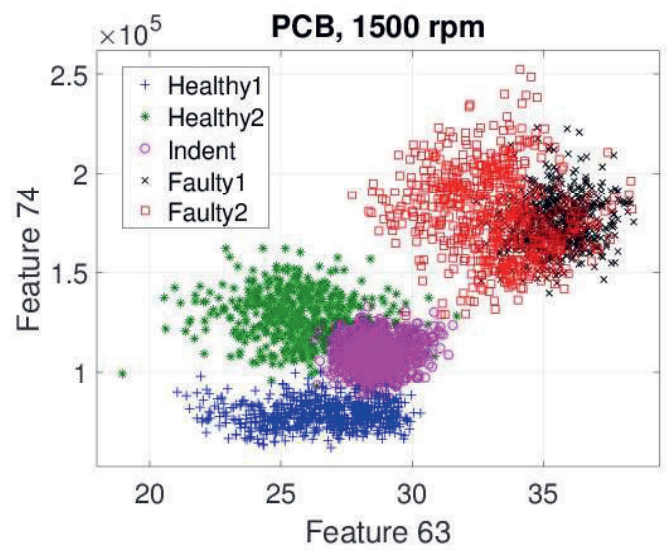

Fig. 10: Scatter plot of top 2 features for the 1500 rpm PCB dataset.

For classification accuracy estimation we trained a quadratic normal Bayes classifier only using observations of the states Healthy2, Indent and Faulty2 (using two 20 second data batches each). After training, validation was performed with another 20 second data batch of all 5 bearing states. However, as target class of the classifier we did not use those five states, but only the simplified states Healthy (Healthy1 and Healthy2), Indent and Faulty (Faulty1 and Faulty2). According to the feature selection step, the $3000 \mathrm{rpm}$ data were validated with the top 3 features and the 1500 rpm data were validated with the top 4 features. The validation yields $99.30 \%$ accuracy for the $3000 \mathrm{rpm}$ data (confusion matrix in Tab. 2) and $82.41 \%$ accuracy (confusion matrix in Tab. 2) for the $1500 \mathrm{rpm}$ data. That result confirms the first conclusions above: the separability of the states Healthy, Indent and Faulty in the $3000 \mathrm{rpm}$ case is satisfying, while the separability is worse in the 1500 rpm case. Especially the state Healthy 1 
is misclassified in the $1500 \mathrm{rpm}$ case. Since Healthy1 was not used for classifier training, it is obviously more likely to be misclassified.

Tab. 1: Confusion matrix for $3000 \mathrm{rpm} P C B$ data and top 3 features.

\begin{tabular}{|c|r|c|c|c|}
\hline \multirow{2}{*}{ Confusion Matrix } & \multicolumn{3}{|c|}{ Estimated State } \\
\cline { 3 - 5 } & Healthy & Ident & Faulty \\
\hline \multirow{4}{*}{$\begin{array}{c}\text { True } \\
\text { State }\end{array}$} & Healthy1 & 192 & 7 & 0 \\
\cline { 2 - 5 } & Healthy2 & 199 & 0 & 0 \\
\cline { 2 - 5 } & Indent & 0 & 199 & 0 \\
\cline { 2 - 5 } & ALT2 & 0 & 0 & 199 \\
\cline { 2 - 5 } & ALT3 & 0 & 0 & 199 \\
\hline
\end{tabular}

Tab. 2: Confusion matrix for $1500 \mathrm{rpm}$ PCB data and top 4 features.

\begin{tabular}{|c|r|c|c|c|}
\hline \multirow{2}{*}{ Confusion Matrix } & \multicolumn{3}{|c|}{ Estimated State } \\
\cline { 3 - 5 } & Healthy & Ident & Faulty \\
\hline \multirow{4}{*}{ True } & Healthy1 & 33 & 166 & 0 \\
\cline { 2 - 5 } State & Healthy2 & 198 & 1 & 0 \\
\cline { 2 - 5 } & Indent & 5 & 194 & 0 \\
\cline { 2 - 5 } & ALT2 & 0 & 0 & 199 \\
\cline { 2 - 5 } & ALT3 & 3 & 0 & 196 \\
\hline
\end{tabular}

To compare the results of the PCB sensor with the MEMS sensor, we extracted the same features from the raw data of the MEMS sensor and validated them in the same way. For the $3000 \mathrm{rpm}$ case that yields in $93.67 \%$ accuracy (confusion matrix in Tab. 3, scatterplot of top 2 features in Fig. 11) and in the $1500 \mathrm{rpm}$ case in $78.99 \%$ accuracy (confusion matrix in Tab. 4, scatterplot of top 2 features in Fig. 12). In both cases, the accuracy of the low-cost MEMS sensor data is lower than the accuracy of the high-end PCB sensor data. However, one might argue that the comparison is not fair, since the feature selection was performed with the PCB data. In our experiments, the MEMS sensor did not perform significantly better when the feature selection was performed using the MEMS data.

Tab. 3: Confusion matrix for $3000 \mathrm{rpm}$ MEMS data and top 6 features.

\begin{tabular}{|c|r|c|c|c|}
\hline \multirow{2}{*}{ Confusion Matrix } & \multicolumn{3}{|c|}{ Estimated State } \\
\cline { 3 - 5 } & Healthy & Ident & Faulty \\
\hline \multirow{4}{*}{ True } & Healthy1 & 199 & 0 & 0 \\
\cline { 2 - 5 } State & Healthy2 & 138 & 61 & 0 \\
\cline { 2 - 5 } & Indent & 2 & 197 & 0 \\
\cline { 2 - 5 } & ALT2 & 0 & 0 & 199 \\
\cline { 2 - 5 } & ALT3 & 0 & 0 & 199 \\
\hline
\end{tabular}

Tab. 4: Confusion matrix for $1500 \mathrm{rpm}$ MEMS data and top 4 features.

\begin{tabular}{|c|r|c|c|c|}
\hline \multirow{2}{*}{ Confusion Matrix } & \multicolumn{3}{|c|}{ Estimated State } \\
\cline { 3 - 5 } & Healthy & Ident & Faulty \\
\hline \multirow{4}{*}{ True } & Healthy1 & 22 & 177 & 0 \\
\cline { 2 - 5 } State & Healthy2 & 199 & 0 & 0 \\
\cline { 2 - 5 } & Indent & 8 & 185 & 6 \\
\cline { 2 - 5 } & ALT2 & 0 & 0 & 199 \\
\cline { 2 - 5 } & ALT3 & 11 & 7 & 181 \\
\hline
\end{tabular}

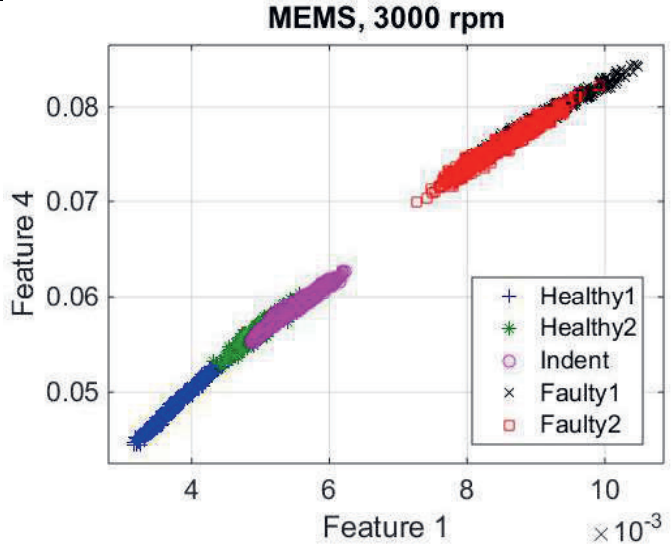

Fig. 11: Scatter plot of top 2 features for the 3000 rpm MEMS dataset.

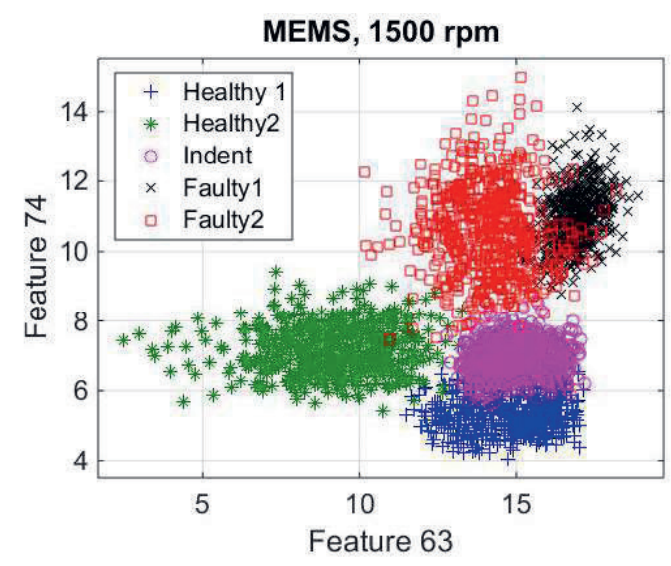

Fig. 12: Scatter plot of top 2 features for the $1500 \mathrm{rpm}$ MEMS dataset.

\section{Conclusions}

The results of this empirical study of vibration based fault diagnosis methods for bearings show that the feature based approach can be used for fault monitoring of bearings. Furthermore it can be concluded that the classification accuracy increases with higher rotational speed of the bearing. The high-end PCB sensor allows higher accuracy values than the low-cost MEMS sensor. The ALT test shows that an upcoming fault can be detected before total failure of the bearing occurs.

The proposed method does not require any parameters or physical information about the system/component to be monitored. This 
makes it very flexible and useful for manifold industrial applications.

\section{Acknowledgments}

This work has been supported by the COMETK2 Center of the Linz Center of Mechatronics (LCM) funded by the Austrian federal government and the federal state of Upper Austria.

This research was partially supported by Flanders Make, the strategic research centre for manufacturing industry.

\section{References}

[1] A. Albabar, S. Mekid, A. Starr, R. Pietruszkiewicz, Suitability of MEMS accelerometers for condition monitoring: An experimental study, Sensors 8(2), 784-799 (2008)

[2] A.P. Ompusunggu, T. Ooijevaar, B. Kilundu, S. Devos, Automated bearing fault diagnostics with cost-effective vibration sensor, In J. Mathew, C. Lim, L. Ma (Eds.), Asset Intelligence through Integration and Interoperability and Contemporary Vibration Engineering Technologies, Proceedings of the 12th World Congress on Engineering Asset Management, 469-472 (2018)

[3] P. Henriquez, J. Alonso, M. Ferrer, C. Travieso, Review of automatic fault diagnosis systems using audio and vibration signals, IEEE Transactions on Systems, Man, and Cybernetics: Systems 44(5), 642-652 (2014)

[4] A. Sait, Y. Sharaf-Eldeen, A Review of Gearbox Condition Monitoring Based on vibration Analysis Techniques Diagnostics and Prognostics, In T. Prolux (Ed.), Rotating Machinery, Structural Health Monitoring, Shock and Vibration 5, Proceedings of the 29th IMAC: A Conference on Structural Dynamics, The Society for Experimental Mechanics, 359-374 (2011)

[5] D. Wang, K. Tsui, Q. Mia, Prognostics and Health Management: A Review of Vibration Based Bearing and Gear Health Indicators, IEEE Access 6, 665-676 (2017)

[6] S. Singh, M. Vishwakarma, A Review of Vibration Analysis Techniques for Rotating Machines, International Journal of Engineering Research \& Technology 3, 757-761 (2015)

[7] M. Jalil, F.A. Butt, A. Malik, Short-time energy, magnitude, zero crossing rate and autocorrelation measurement for discriminating voiced and unvoiced segments of speech signals, The International Conference on Technological Advances in Electrical, Electronics and Computer Engineering, 208$212(2013)$

[8] V. Sharma, A. Parey, A review of gear fault diagnosis using various condition indicators, Procedia Engineering 144, 253-263 (2016)

[9] P.D. McFadden, M.M. Toozhy, Applications of Synchronous Averaging to Vibration Monitoring of Rolling Element Bearings, Mechanical
Systems and Signal Processing 14(6), 891-906 (2000)

[10] H. Heidari Bafroui, A. Ohadi, Application of wavelet energyband Shannon entropy for feature extraction in gearbox fault detection under varying speed conditions, Neurocomputing 133, 437-445 (2014)

[11] Y. Lei, Z. He, Y. Zi, Q. Hu, Fault diagnosis of rotating machinery based on multiple ANFIS combination with GAs, Mechanical Systems and Signal Processing 21, 2280-2294 (2007)

[12] C. Shen, D. Wang, F. Kong, P.W. Tse, Fault diagnosis of rotating machinery based on the statistical parameters of wavelet packet paving and a generic support vector regressive classifier, Measurement 46, 1551-1564 (2013)

[13] R: Bajric, N. Zuber, G.A. Skrimpas, N. Mijatovic, Feature Extraction Using Discrete Wavelet Transform for Gear Fault Diagnosis of Wind Turbine Gearbox, Shock and Vibration (2016)

[14] J. Antoni, R.B. Randall, The spectral kurtosis: application to the vibratory surveillance and diagnostics of rotating machines, Mechanical Systems and Signal Processing 20, 308-331 (2006)

[15] F.d.A. Boldt, T.W. Rauber, F.M. Varejao, Feature Extraction and Selection for Automatic Fault Diagnosis of Rotating Machinery (2013)

[16] H. Konstantin-Hansen, H. Herlufsen, Envelope and Ceptstrum Analyses for Machinery Fault Identification, Sound \& Vibration, 10-12 (2010)

[17] I. Guyon, A. Elisseeff, An introduction to variable and feature selection, Journal of Machine Learning Research 3, 1157-1182 (2003)

[18] J.G: Dy, C.E. Brodley, Feature Selection for Unsupervised Learning, Journal of Machine Learning Research 5 (2004)

[19] G.J. McLachlan, Mahalanobis distance, Resonance 4(6), 20-26 (1999)

[20] D. de Ridder, D.M.J. Tax, B. Lei, G. Xu, M. Feng, Y. Zou, F. can der Heijden, Classification, Parameter Estimation and State Estimation: An Engineering Approach Using MATLAB, John Wiley \& Sons, Ltd. (2017)

[21] D. Tax, One-class classification, PhD thesis, Delft University of Technology (2001)

[22] J. Halme, P. Anderson, Rolling contact fatigue and wear fundamentals for rolling bearing diagnostics - state of the art, Journal of Engineering Tribology, 377-393 (2009)

[23] D.M. Hawkins, D.H. Olwell, Cumulative Sum Charts and Charting for Quality Improvement, Springer (1998) 